\title{
Highly uniform and low-loss passive silicon photonics devices using a 300mm CMOS platform
}

\author{
Shankar Kumar Selvaraja ${ }^{1}$, Peter De Heyn ${ }^{2}$, Gustaf Winroth ${ }^{1}$, Patrick Ong ${ }^{1}$, Guy Lepage ${ }^{1}$, Celine Cailler ${ }^{3}$, \\ Arnaud Rigny $^{3}$, Konstantin K. Bourdelle ${ }^{3}$, Wim Bogaerts ${ }^{2}$, Dries Van Thourhout ${ }^{2}$, Joris Van Campenhout ${ }^{1}$, \\ and Philippe Absil ${ }^{1}$ \\ ${ }^{1}$ imec, Leuven, Belgium, ${ }^{2}$ Ghent University-imec, Ghent, Belgium, ${ }^{3}$ Soitec, Bernin, France \\ shankar.selvaraja@imec.be
}

\begin{abstract}
Using an advanced 300mm CMOS-platform, we report record-low and highly-uniform propagation loss: $0.45 \pm 0.12 \mathrm{~dB} / \mathrm{cm}$ for wires, and $2 \mathrm{~dB} / \mathrm{cm}$ for slot waveguides. For WDM devices, we demonstrate channel variation $(3-\sigma)$ within-wafer and within-device of $6.1 \mathrm{~nm}$ and $1.2 \mathrm{~nm}$ respectively.

OCIS codes: $130.3120,230.7370,230.7390,140.4780,060.1810$
\end{abstract}

\section{INTRODUCTION}

Next-generation silicon photonics transceivers will require the integration of ultra-low-power active devices with ultra-low-loss passive devices enabling wavelength-division multiplexing (WDM). A major challenge in the manufacturing of such devices is the extreme sensitivity of the device and waveguide properties (phase and amplitude transfer control) on the cross-sectional dimensions and sidewall quality of the employed photonic wire and rib structures, which results in poor device uniformity across a die or a wafer [1].

In this paper, we report on a significant improvement in propagation loss well below $1 \mathrm{~dB} / \mathrm{cm}$ with a $1-\sigma$ variation of $0.12 \mathrm{~dB} / \mathrm{cm}$ across a $300 \mathrm{~mm}$ for a single-mode $400 \mathrm{~nm}$ x $220 \mathrm{~nm}$ wire waveguide. For WDM devices, an absolute channel wavelength $3-\sigma$ variation of $6.1 \mathrm{~nm}$ is demonstrated across a wafer, as well as a channel-spacing 3- $\sigma$ variation of $1.2 \mathrm{~nm}$. Furthermore, for the first time we verify the wide belief that silicon photonic device and circuit performance and variability will be greatly improved with advanced CMOS processes and substrate technology.

\section{FABRICATION TECHNOLOGY}

For the device fabrication, we used a $300 \mathrm{~mm}$ Silicon-On-Insulator (SOI) wafer with $220 \mathrm{~nm}$ of crystalline Si top layer and 2000nm buried oxide (BOX). The SOI wafers were manufactured by Soitec using SmartCut ${ }^{\mathrm{TM}}$ technology. Fig.1 depicts a typical Si thickness distribution. The wafers show a high degree of uniformity with 3- $\sigma$ variation of $1.65 \mathrm{~nm}(0.75 \%)$ and a wafer range of $2.5 \mathrm{~nm}(1.1 \%)$. For the device patterning, we used a modified 28-nm shallowtrench isolation (STI) process [2]. The pattern on the photomask is transferred into silicon using 193nm immersion lithography and a dry etch process. We use two etch levels, a $220 \mathrm{~nm}$ deep level for waveguide definition and $70 \mathrm{~nm}$ shallow level for fiber-chip grating coupler definition. After dry etch and resist removal, the waveguides were covered with silicon dioxide and planarized. After planarization the wafers are tested optically.

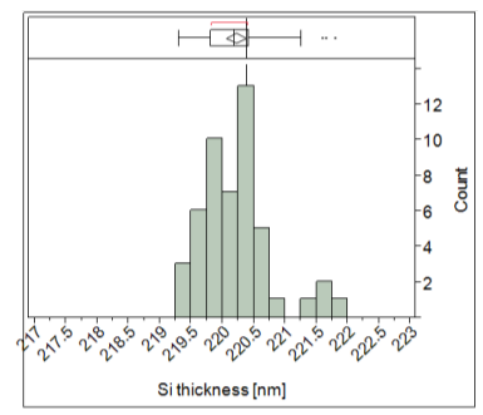

Fig. 1 Si thickness distribution over a 300mm SOI wafer.

\section{LOW-LOSS WAVEGUIDES}

We use two types of waveguides to demonstrate the loss performance: (1) single-mode photonic full-depth etched wires and (2) slot waveguide. The propagation loss in these waveguides is mainly attributed to light scattering off the etched sidewalls. Therefore, losses are a direct qualification of the patterning process quality. It has been shown in [3] that low roughness patterning can be achieved by using 193immersion lithography and an optimized dry etch 
process. To assess the propagation loss, we fabricated spiral photonic wire waveguides of three different widths (400, 450 and $500 \mathrm{~nm})$ and slot waveguide (260nm rail width, $100 \mathrm{~nm}$ slot width). Each waveguide comes in 4 lengths $(1,2,4$ and $7 \mathrm{~cm})$ from which the propagation loss is extracted by a linear fit. The bend radius of wires and slot waveguides was $10 \mu \mathrm{m}$ and $20 \mu \mathrm{m}$ respectively.

In the $C$ band, we measure an average propagation loss of $0.8,0.74$ and $0.5 \mathrm{~dB} / \mathrm{cm}$ for 400,450 and $500 \mathrm{~nm}$ wide wire waveguides respectively over a $300 \mathrm{~mm}$ wafer (Fig.2). The error margin on individual data point is $<0.02 \mathrm{~dB}$. To our knowledge, this is the lowest loss reported for fully etched single-mode silicon wire. The waveguide losses are verified across the $300 \mathrm{~mm}$ wafer with $3-\sigma$ variation of $0.12 \mathrm{~dB} / \mathrm{cm}$ and a range of $0.12 \mathrm{~dB} / \mathrm{cm}$ (Fig. 3). This represents over $50 \%$ improvement as compared to the best single-mode waveguide reported loss on a $200 \mathrm{~mm}$ platform.

For slot waveguides, we measure a propagation loss of $2 \mathrm{~dB} / \mathrm{cm}$ at $1550 \mathrm{~nm}$ (Fig. 4), while with the $200 \mathrm{~mm}$ platform, a similar type of waveguide geometry yields a loss of between $10-15 \mathrm{~dB} / \mathrm{cm}$.

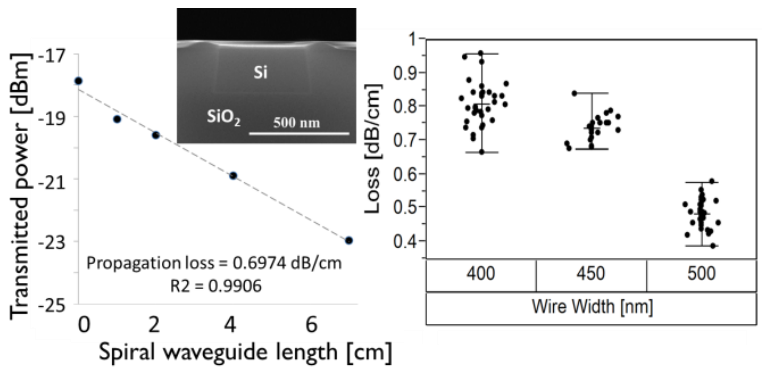

Fig. 2. Propagation loss extraction of a 450nm photonic wire. Loss of different waveguide widths. Inset shows a typical cross-section of a $450 \mathrm{~nm}$ wide wire.

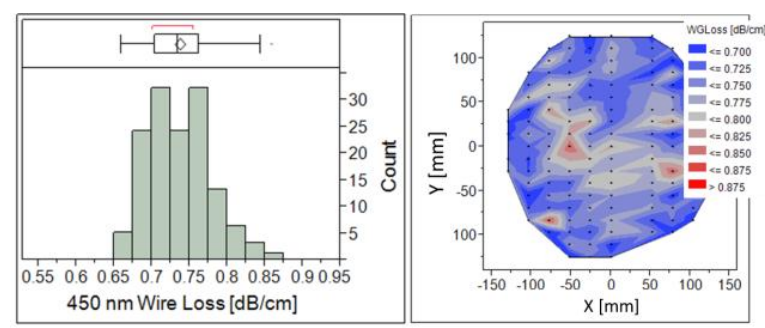

Fig. 3. Within wafer propagation loss uniformity of a $450 \mathrm{~nm}$ wire waveguide.
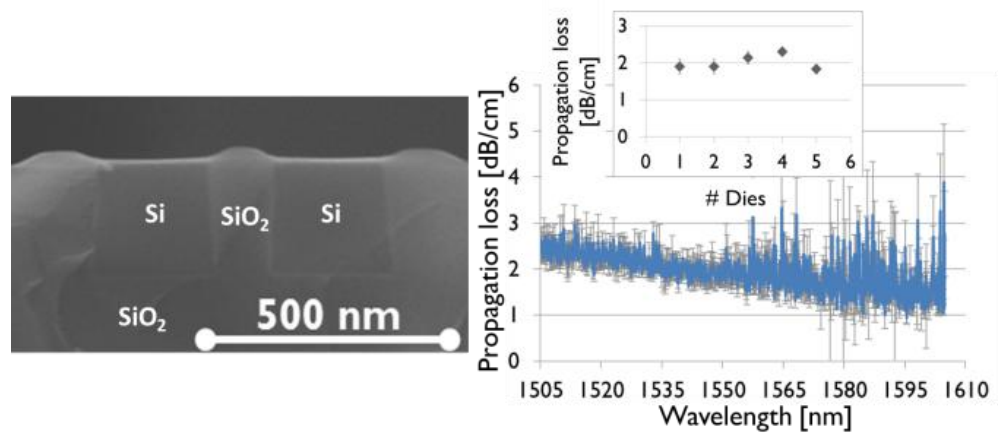

Fig. 4 (left)Cross-section SEM image of a slot waveguide and (right) typical transmission spectrum and loss spectrum.

Fig. 6 shows the distribution of the drop wavelength of the first channel across the $300 \mathrm{~mm}$ wafer. We observe a within-wafer $3 \sigma$ variation of $6.1 \mathrm{~nm}$, which agrees well with the waveguide width variability over the wafer, where $3-\sigma$ variation of $7.65 \mathrm{~nm}$ is observed.

Fig. 7 shows the variability $(1-\sigma)$ of the CS error for all channels across a $200 \mathrm{~mm}$ and a $300 \mathrm{~mm}$ wafer, as a function of the average CS. By using the $300 \mathrm{~mm}$ platform the $1-\sigma$ variability of the CS error is reduced to $0.4 \mathrm{~nm}$ (at an average CS of $2.5 \mathrm{~nm}$ ) from $0.7 \mathrm{~nm}$ in the $200 \mathrm{~mm}$ platform (at an average CS of $2.55 \mathrm{~nm}$ ) [4]. 


\section{CONCLUSION}

We presented a highly uniform and low-loss silicon photonics platform using advanced 300mm CMOS fabrication technology. The substantial improvement in propagation loss and WDM device matching over the wafer is an important step for realizing next-generation low-power and high-density silicon photonics devices and systems.

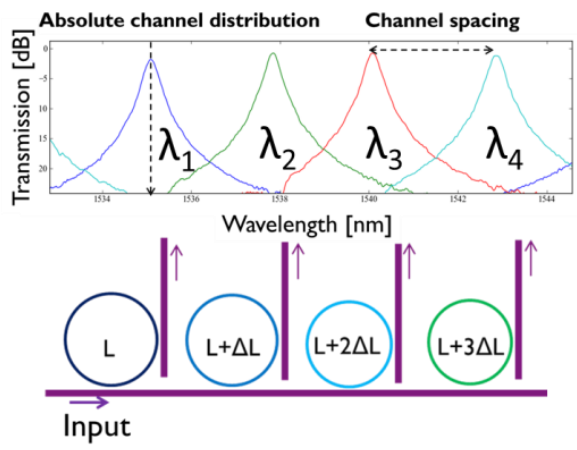

Fig. 5 (left)Schematic of $1 \times 4$ single-ring de-multiplexer. Right) Typical spectral response and the parameters studied.

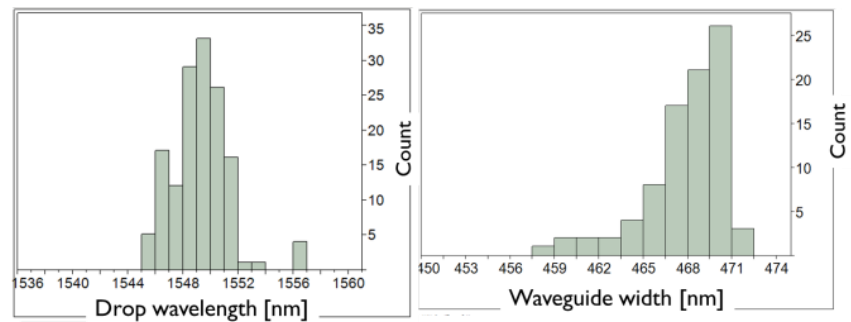

Fig. 6 (left) Absolute channel variability of one of the channels, (right) 450nm photonic wire distribution over a $300 \mathrm{~mm}$ wafer

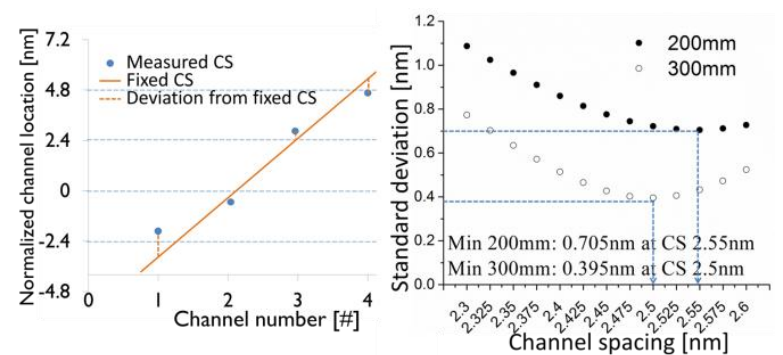

Fig. 7 (Left)Schematic of channel spacing and deviation analysis. (Right) Variability of channels spacing between 200mm and 300mm platform.

\section{ACKNOWLEDGEMENT}

The authors wish to thank the imec p-line for the fabrication of the silicon photonics wafers. This work was supported by imec's Core Partner Program and the ERC-project ULPPIC. The research leading to these results has received funding from the European Community's Seventh Framework Programme (FP7/2007-2013) under grant agreement n 318178-PLAT4M.

\section{REFERENCE}

[1] Krishnamoorthy, A. V., Pinguet, T., Mekis, A., Thacker, H., Shubin, I., Raj, K., and Cunningham, J. E, “Exploiting CMOS Manufacturing to Reduce Tuning Requirements for Resonant Optical Devices", IEEE Photo. J., 3(3), 567-579 (2011).

[2] Shankar Selvaraja, Gayle Murdoch, Alexey Milenin, Christie Delvaux, Patrick Ong, Shibnath Pathak, Diedrik Vermeulen, Gunther Sterckx, Gustaf Winroth, Peter Verheyen, Guy Lepage, Wim Bogaerts, Roel Baets, Joris Van Campenhout, Philippe Absil, "Advanced 300-mm waferscale patterning for silicon photonics devices with record low loss and phase errors", OECC2012 PD15-16 (2012).

[3] Hody, H., Vecchio, E., Boullart, W., \& Boullart, W., "Double patterning with dual hard mask for 28-nm node devices and below.", J. Micro/Nanolith. MEMS MOEMS 12(4), 041306 (2013).

[4] Peter De Heyn, Jeroen De Coster, Peter Verheyen, Guy Lepage, Marianna Pantouvaki, Philippe Absil, Wim Bogaerts, Joris Van Campenhout, and Dries Van Thourhout, "Fabrication-Tolerant Four-Channel Wavelength-Division-Multiplexing Filter Based on Collectively Tuned Si Microrings" J. Lightw. Technol. 31(16), 2785 - 2792 (2013). 